\title{
Modeling of Immunosensors under Nonequilibrium Conditions
}

\author{
I. Mathematic Modeling of Performance Characteristics'
}

\author{
Se-Hwan Paek* and Willfried Schramm*, \\ *The University of Michigan, Reproductive Sciences Program and Bioengineering Program, 300 North Ingalls, Ann Arbor, \\ Michigan 48109; and †BioQuant, Inc., 1919 Green Road, Ann Arbor, Michigan 48105
}

Received December 17, 1990

Immunosensors for the detection of small analytes that use analyte-enzyme conjugates as signal generators require special attention if operated under nonequilibrium conditions. If the size of the analyte and the analyte-enzyme conjugate differ substantially, the two antigens do not diffuse at the same rate. This can cause time-dependent shifts in the sensitivity of competitive immunoassays. Therefore, immunosensors operating at short incubation times require precise timing that meets closely the specifications for which the sensors were calibrated. As an example, we have analyzed kinetic binding curves for the quantitative determination of progesterone with an immobilized monoclonal antibody and a conjugate between horseradish peroxidase and progesterone as signal generator. Mathematical paradigms have been developed to simulate the diffusion, antigen-antibody complex formation, and competitive binding processes in this analytical system. Doseresponse curves obtained under nonequilibrium conditions can vary substantially from those obtained at equilibrium of antigen-antibody interaction. The degree of this variation depends on the performance characteristics of the major components of the immunosensor. The developed mathematical solutions reflect experimental results and can be used to model optimal conditions for immunosensors operating under nonequilibrium conditions. In this paper (Part I), we report on the mathematical modeling of the interaction between analyte, analyte-enzyme conjugate, and an immobilized antibody. In Part II (W. Schramm and S.-H. Paek (1991) Anal. Biochem. 196), we present experimental results and compare them with the theoretical models. (c) 1991 Academic Press, Inc.
In the area of sensor technology, one of the performance characteristics is the "response time." For many sensors, a fast response time is desirable. Obviously, the definition of "fast" is relative, depending on the particular problem to be solved by a measurement and on the technique used for analyte detection and signal generation by a sensor. Recent developments in sensor technology $(1,2)$ use biomolecules for the detection and quantitative determination of analytes in solutions. In physiological systems, the natural function of biomolecules is to control regulatory mechanisms that extend frequently over time periods of minutes or even hours. However, their often inherent slow response times can cause limitations if used in biosensors.

One class of hiomolecules used in sensors designed to detect complex organic molecules is the immunoglobulin (immunosensors $(3,4)$ ). With the immunoassay technique that forms the basis for immunosensors, incubation times of several hours, even days, are not uncommon. The antigen-antibody complex formation (a) is a reversible process and (b) obeys an equilibrium reaction that follows the law of mass action. The number of antigen molecules bound to immunoglobulins increases asymptotically over time to reach a state at which the number of molecules associating with the antibodies and those molecules dissociating from the antibodies are equal. Many quantitative determinations with immunoglobulins are performed at this equilibrium state.

However, immunoglobulins can be used in the non-

\footnotetext{
' Part II: Experimental Determination of Performance Characteristics.
} 
equilibrium state to reduce response times in immunosensors. In this communication (Part I), we outline some of the problems to be considered for the engineering of immunosensors operating under nonequilibrium conditions, and we also present solutions for calculating the performance of the sensors under these conditions. The theoretical determination of kinetic variables using mathematical models permits the appropriate selection of immunoglobulins and the synthesis of analyte-enzyme conjugates with optimal binding characteristics. In a subsequent paper (Part II) (5), we analyze how the models predict experimental results.

\section{ANALYTE-ENZYME CONJUGATE AS SIGNAL GENERATOR}

For the majority of analytical systems that use immunoglobulins as bioreceptors, binding of an antigen to an antibody cannot be directly monitored because the resulting complex does not usually provide a suitable physical signal. Therefore, for many applications, "labels" are used for signal generation. For many years, radioisotopes have been the most popular labels but these are often replaced today by other molecules such as luminescent and fluorescent markers. One of the most commonly used labels is enzymes. Enzymes can generate either a photometrically or an electrochemically detectable signal. We will investigate the kinetic reactions with enzymes as signal generators and we have selected the competitive immunoassay as an example. Similar considerations might apply for other types of antigenantibody binding reactions used for the construction of biosensors. We have used a solid-phase immunoassay as a model which is probably representative for most immunosensors. In a solid-phase assay, one of the components participating in the analytical reaction is immobilized on a solid matrix, e.g., on an electrode or an optic fiber. For the investigated example, the immunoglobulin is immobilized.

If an enzyme-labeled antigen is used for signal generation in immunosensors, the size of the label can substantially differ from that of the native antigen (analyte). This has consequences for the kinetics of the different binding reactions. A competitive immunoassay as shown in Fig. 1 implies that the analyte competes with the analyte-enzyme conjugate for binding sites on the antibody. This happens only if the two species are reasonably equally recognized by the antibody. Provided that is the case, binding over time for the native antigen is different than for the enzyme-labeled antigen if their molecular masses vary substantially because the smaller molecule diffuses faster to the immobilized antibody than does the larger conjugate. Therefore, we have a sequential immunoassay where one of the two competing components (i.e., the analyte) reaches the antibody first to initiate the binding reaction that culmi-

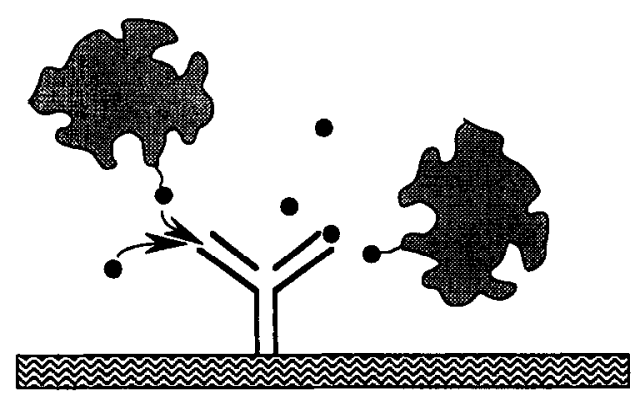

FIG. 1. Competitive binding assay. Analyte (dots) from the sample competes with the enzyme-labeled antigen for binding sites on the antibody (immobilized on a solid matrix). If the amount of antibody is constant and limited (with regard to the antigen), and a constant amount of enzyme-labeled antigen is added, the amount of analyte in the sample determines the quantity of enzyme label bound to the antibody. Thus, the signal generated from the enzyme label is inversely proportional to the amount of analyte in the sample.

nates in the equilibrium state where the on-rate and the off-rate for the analyte-antibody complex formation are equal. The other antigen (the larger conjugate) reaches equilibrium later. It is the combination of these two superimposed, temporarily delayed equilibrium reactions that determines the performance characteristics of the immunosensor.

This is of little consequence if the incubation time is long enough to permit both antigen species to reach binding equilibrium. However, if the immunosensor is operated under nonequilibrium conditions to reduce the response time, the different kinetics of the two binding reactions have to be taken into consideration for calibration purposes. We have made an attempt to model the response time of the described type of immunosensor under nonequilibrium conditions.

\section{THEORETICAL DETERMINATION OF KINETIC VARIABLES}

Many performance characteristics of immunosensors can be predicted by theoretical models. In this section, we introduce the mathematical model for the theoretical determination of kinetic variables and present two approaches to calculate the binding of antigen to antibody over time. However, to execute the calculations according to the model, a minimum of independent variables need to be experimentally determined (see Part II). This is comparatively simple for variables derived from labeled antigens since these are directly generating a measurable signal. The native antigen, however, cannot be measured directly in many designs of immunosensors and some kinetic parameters need to be indirectly determined.

\section{Mathematical Model}

For the development of the mathematical model we have selected the geometry of a microwell (Fig. 2). An 


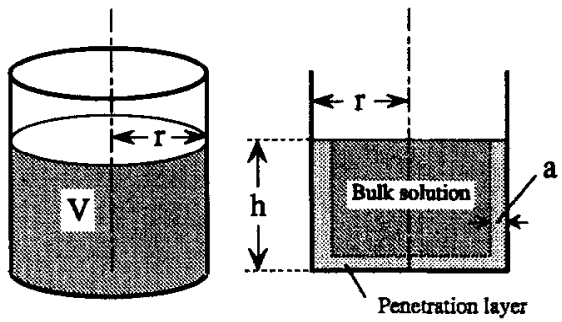

FIG. 2. Geometry of a microwell. For the definition of the symbols see Table 1.

antibody is chemically bound to a surface area contacted by $200 \mu \mathrm{l}$ of medium. These same conditions were used for the validation of the theoretical predictions by experimental studies. The symbols and dimensions used for the model are described in Table 1.

Diffusion of molecules and binding of antigens to the immobilized antibody at the liquid-solid interface are the two processes that are mathematically described.

Differential rate equation for diffusion. If an immunosensor with an immobilized antibody on the surface is exposed to the solution that contains the analyte to be measured, a concentration gradient of the analyte (antigen) ensues. The analyte is removed from the bulk solution by binding to the antibody. As a dynamic equilibrium process, the antigen-antibody binding rate is determined by the on- and off-rate, i.e., the association of the antigen with the antibody and the dissociation from the antigen-antibody complex. The question arises: Is the approach to equilibrium limited by the ability of the antigen molecules to reach the antibody on the solid surface? In other words, is the establishment of equilibrium diffusion limited? In practical terms, the investigator wants to know if a certain design of an immunosensor can be operated with or without agitation. Diffusion of reagents on solid-liquid interfaces of microwells toward the formation of antigen-antibody complexes without agitation was previously described (6). We are theoretically investigating the diffusion processes under agitation. In Part II of this study, we describe the experimental results for diffusion processes under agitation.

Diffusion is the transport of a constituent from a region of higher concentration to that of a lower concentration. This process consists of three components which are described in the context of an immunosensor with an immobilized antibody on a solid surface and a binding reaction at the liquid-solid interface (for review see (7)):

1. Convective diffusion, the movement of molecules (or particles) from the bulk solution to the surface. This is a three-dimensional process $(8,9)$.

2. Lateral diffusion, the movement of molecules on the surface of the solid phase toward an immobilized immunoglobulin. This is a two-dimensional process $(10,11)$.

3. Rotational diffusion, to direct the antigen spatially toward the idiotypic site of the immunoglobulin so that

TABLE 1

Symbols and Their Descriptions Used for the Theoretical Determination of Kinetic Variables by Mathematical Models

\begin{tabular}{|c|c|c|}
\hline Symbol & Description & Dimension \\
\hline$a$ & Thickness of penetration layer & $\mathrm{mm}$ \\
\hline$[\mathrm{Ab}]_{\mathrm{f}},[\mathrm{Ab}]_{\mathrm{t}}$ & $\begin{array}{l}\text { Density of binding sites of antibody on the solid surface (subscript } f \text { stands for unoccupied } \\
\text { and } t \text { for total binding sites) }\end{array}$ & $\mathrm{mol} \mathrm{mm}^{-2}$ \\
\hline$[\mathrm{Ag}]_{\mathrm{b}},[\mathrm{Ag}]_{\mathrm{g}},[\mathrm{Ag}]_{\mathrm{t}}$ & Concentration of antigen (subscript $b$ in bulk solution, $s$ on solid surface, and $t$ for total) & $\mathrm{mol} \mathrm{mm}^{-3}$ \\
\hline$[\mathrm{Ab}: \mathrm{Ag}]$ & Density of antigen-antibody binding complex & $\mathrm{mol} \mathrm{mm}^{-2}$ \\
\hline$B$ & Amount of antigen bound to antibody & $\mathrm{mol} /$ well \\
\hline$C$ & Defined by $N_{\mathrm{Da}} k_{c}\{(1+2 \lambda) /(r \lambda)\}$ & \\
\hline$D$ & Effective diffusion coefficient of antigen & $\mathrm{mm}^{2} \mathrm{~s}^{-1}$ \\
\hline$h$ & Height of liquid head in microwell & $\mathbf{m m}$ \\
\hline$J_{\text {in }}, J_{\text {out }}$ & Influx and efflux across a defined boundary & 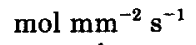 \\
\hline & Convective mass transfer coefficient $(D / a)$ & $\mathrm{mm} \mathrm{s}^{-1}$ \\
\hline$k_{\text {off }}$ & Off-rate constant of antigen from antigen-antibody complex & $\mathrm{s}^{-1}$ \\
\hline & On-rate constant of antigen for antigen-antibody complex formation & $\mathrm{mm}^{3} \mathrm{~mol}^{-1} \mathrm{~s}^{-1}$ \\
\hline$\left[N_{\mathrm{Ag}}\right]_{\mathrm{b}},\left[N_{\mathrm{Ag}}\right]_{\mathrm{p}}$ & Amount of antigen in bulk solution (b) and penetration layer (p) & mol \\
\hline$N_{\mathrm{Da}}$ & Dimensionless Damkoehler number $\left(k_{\mathrm{on}}[\mathrm{Ab}]_{\mathrm{t}} / k_{\mathrm{c}}\right)$ & \\
\hline$r$ & Inner radius of microwell & $\mathbf{m m}$ \\
\hline$S$ & Surface area of microwell that is covered with liquid & $\mathrm{mm}^{2}$ \\
\hline$t$ & Time & $\mathbf{s}$ \\
\hline$T$ & 'Total amount of antigen & $\mathrm{mol} /$ well \\
\hline$T^{\prime}$ & Total amount of binding sites & $\mathrm{mol} / \mathrm{well}$ \\
\hline$V$ & Volume of liquid in microwell & $\mathrm{mm}^{3}$ \\
\hline$\lambda$ & Ratio of $h / r$ in microwell & \\
\hline
\end{tabular}




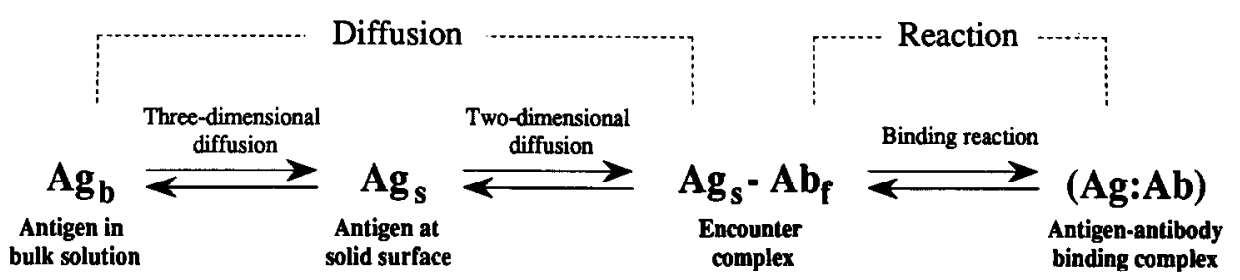

FIG. 3. Diffusion and reaction processes that are involved in leading to the formation of the antigen-antibody complex. The two-dimensional diffusion consists of lateral and rotational diffusion (see text). The three diffusion processes result in the formation of the encounter complex which is the precondition for the formation of the antigen-antibody complex.

binding can occur. This is also a two-dimensional process (12).

These three diffusion processes lead to the establishment of an encounter complex which is the precondition for the antigen-antibody complex. The relative rate of formation of these two complexes indicates if the sensor is diffusion controlled or reaction controlled.

It is difficult to measure the diffusion processes separately, but the overall (effective) diffusion coefficient can be experimentally determined. Alternatively, the effective diffusion coefficient can be calculated if the molecular dimensions of the antigen(s) participating in the antigen-antibody reaction are known. However, these calculations are complicated by the effect that repulsive forces (e.g., charge interactions) and nonsymmetric configurations of molecules in solution and on the surface can have on the diffusion of molecules.

For this model, we have not taken into consideration the surface charge of the antigen and the surface area. The electrostatic forces between the surface and antigen is inversely proportional to the distance. Therefore, the three-dimensional diffusion is not substantially affected by charges. The antigen present near the solid surface may interact with the charged surface, the strength of repulsion or attraction depending on the charge density. These forces can affect the lateral diffusion rate (13).

Three-dimensional diffusion can be accelerated by agitating the incubation mixture so that molecules reach the surface faster. However, even with most vigorous agitation, this type of diffusion can never be completely abolished. Near the surface, a layer of water and medium components forms that is organized by electrostatic forces resulting from the interaction of the surface and the components of the medium, including the diffusive substance (i.e., antigen). This is called the penetration layer or hydrodynamic layer ( $a$ in Fig. 2 and Table $1 ;(8)$ ). The diffusive substance may only marginally affect the thickness of the penetration layer if it is vastly outnumbered by other medium components (e.g., gelatin used in incubation buffers).

The formation of the encounter complex (Fig. 3) finishes the process of diffusion and initiates the formation of the antigen-antibody complex, for which the kinetics are determined by the reaction rate ( $k_{\mathrm{on}}$ and $\left.k_{\mathrm{off}}\right)$.

Equations for two of the three other diffusion rates are difficult to formulate (lateral and rotational). Therefore, we developed an equation only for three-dimensional mass transfer [9]. This expression requires the diffusion coefficient, $D$, for the solution $\left(k_{\mathrm{c}}=D / a\right)$. However, we entered into [9] the effective diffusion coefficient (i.e., the sum of the three diffusion processes). Three-dimensional mass transfer seems to contribute the largest component in $D$ [9] so that potential deviations in the following calculations are negligible. This was finally confirmed by the good correlation of the theoretically developed and the experimentally determined data for kinetic binding curves (Part II).

In Eqs. [1] to [9] we explain the formation rate of the encounter complex (Fig. 3). Although the microwell has the geometry of a cylinder, we will treat all surfaces as one plane. The thickness of the penetration layer for diffusion ( 8 to $9 \times 10^{-2} \mathrm{~mm}$ ) is much smaller than the radius of the microwell $(3.3 \mathrm{~mm})$. Since we are working under stirring conditions, molecules in the bulk solution are uniformly distributed and diffusion takes place only in the thin penetration layer that is at first approximation independent of the well geometry. The efflux of antigen from the bulk solution equals the influx into the penetration layer, while the efflux from the penetration layer reflects binding to the antibody, i.e., withdrawal of the antigen from the system. Two material balance equations for the penetration layer [5] and the bulk solution [8] are developed and then combined to obtain the diffusion rate that reflects the formation of the encounter complex over time [9].

For the determination of the concentration of antigen in the penetration layer, the influx of antigen from the bulk solution is proportional to the diffusion coefficient $(D)$ and the concentration gradient $\left(\left\{[\mathrm{Ag}]_{\mathrm{b}}-[\mathrm{Ag}]_{\mathrm{s}}\right\} / a\right)$ (Nernst equation (8)).

$$
J_{\text {in }}=(D / a)\left([\mathrm{Ag}]_{\mathrm{b}}-[\mathrm{Ag}]_{\mathrm{g}}\right)=k_{\mathrm{c}}\left([\mathrm{Ag}]_{\mathrm{b}}-[\mathrm{Ag}]_{\mathrm{s}}\right) .
$$

The efflux of free antigen (withdrawal from the diffusion process) by binding to the immobilized antibody 
follows the binding rate (based on a monovalent binding of antigen to antibody):

$$
J_{\text {out }}=k_{\text {on }}[\mathrm{Ab}]_{\mathrm{f}}[\mathrm{Ag}]_{\mathbf{s}}-k_{\text {off }}[\mathrm{Ab}: \mathrm{Ag}] .
$$

To calculate the accumulation of free antigen molecules in the penetration layer, we estimate an average concentration derived from that of free antigen on the solid surface and that found in the bulk solution (assuming a linear gradient in the penetration layer).

$$
d\left[N_{\mathrm{Ag}}\right]_{\mathrm{p}} / d t=d\left\{a S\left([\mathrm{Ag}]_{\mathrm{b}}+[\mathrm{Ag}]_{\mathrm{s}}\right) / 2\right\} / d t .
$$

The establishment of a linear gradient in the penetration layer in a model similar to that discussed here has been described in detail $(6,8,14)$. Under stirring conditions, the antigen molecules are distributed in a linear concentration gradient within the order of seconds after addition of antigen to the wells. This compares to times of $>30 \mathrm{~min}$ to hours for reaching the state of equilibrium between immobilized antibody and antigen.

By applying the conservation law of mass (accumulation $=$ influx - efflux $)$ we obtain

$$
d\left[N_{\mathrm{Ag}}\right]_{\mathrm{p}} / d t=\left(J_{\text {in }}-J_{\text {out }}\right) S .
$$

Inserting Eqs. [1], [2], and [3] into [4], the sum of the change of concentration of the antigen in the bulk solution and on the solid surface is

$$
\begin{array}{r}
d[\mathrm{Ag}]_{\mathrm{b}} / d t+d[\mathrm{Ag}]_{\mathrm{s}} / d t=(2 / a)\left\{k_{\mathrm{c}}\left([\mathrm{Ag}]_{\mathrm{b}}-[\mathrm{Ag}]_{\mathrm{s}}\right)\right. \\
\left.-\left(k_{\mathrm{on}}[\mathrm{Ab}]_{\mathrm{f}}[\mathrm{Ag}]_{\mathrm{s}}-k_{\mathrm{off}}[\mathrm{Ab}: \mathrm{Ag}]\right)\right\} .
\end{array}
$$

For the bulk solution, the net flux across the boundary between bulk solution and penetration layer [6] and accumulation within the bulk solution [7] are

$$
\begin{aligned}
J_{\text {in }}-J_{\text {out }} & =-k_{\mathrm{c}}\left([\mathrm{Ag}]_{\mathrm{b}}-[\mathrm{Ag}]_{\mathrm{g}}\right) \\
d\left[N_{\mathrm{Ag}_{\mathrm{b}}}\right]_{\mathrm{b}} / d t & =d\left\{[\mathrm{Ag}]_{\mathrm{b}}(V-a S)\right\} / d t .
\end{aligned}
$$

Equations [6] and [7] are combined

$$
d[\mathrm{Ag}]_{\mathrm{b}} / d t=-\left\{k_{\mathrm{c}} S /(V-a S)\right\}\left([\mathrm{Ag}]_{\mathrm{b}}-[\mathrm{Ag}]_{\mathrm{s}}\right) .
$$

Inserting Eq. [8] into [5], the final differential equation for the diffusion rate under stirring conditions is

$$
\begin{aligned}
d[\mathrm{Ag}]_{\mathrm{s}} / d t=k_{\mathrm{c}}\{(2 / a)+S /(V-a S)\}\left([\mathrm{Ag}]_{\mathrm{b}}-[\mathrm{Ag}]_{\mathrm{s}}\right) \\
-(2 / a)\left(k_{\mathrm{on}}[\mathrm{Ab}]_{\mathrm{f}}[\mathrm{Ag}]_{\mathrm{s}}-k_{\mathrm{off}}[\mathrm{Ab}: \mathrm{Ag}]\right) .
\end{aligned}
$$

The concentration of antigen that reaches the surface by diffusion, $[\mathrm{Ag}]_{\mathrm{g}}$, represents that of the encounter complex which is the precursor for antigen-antibody complex formation.

Equation for antigen-antibody binding reaction. The rate of formation of antigen-antibody complex, [Ab:Ag], (reaction rate) is a function of the concentrations of the unoccupied binding sites, $[\mathrm{Ab}]_{\mathrm{r}}$; the antigen at solid surface, $[\mathrm{Ag}]_{\mathrm{B}}$; and the antigen-antibody binding complex, [Ab:Ag].

$$
d[\mathrm{Ab}: \mathrm{Ag}] / d t=k_{\text {on }}[\mathrm{Ab}]_{\mathrm{f}}[\mathrm{Agg}]_{\mathrm{s}}-k_{\text {off }}[\mathrm{Ab}: \mathrm{Ag}] .
$$

Equations for material balance (law of mass action). To solve Eqs. [9] and [10] for the four unknown variables $\left([\mathrm{Ag}]_{b},[\mathrm{Ag}]_{\mathrm{s}},[\mathrm{Ab}]_{\mathrm{f}}\right.$, and $\left.[\mathrm{Ab}: \mathrm{Ag}]\right)$, two additional equations are required. These are provided by the material balance equations for the antigen and the antibody binding sites.

The total molar amount of antigen is the sum of the molar amount in the bulk solution, in the penetration layer, and the amount bound to antibody:

$$
\begin{aligned}
V[\mathrm{Ag}]_{\mathrm{t}}=( & V-a S)[\mathrm{Ag}]_{\mathrm{b}} \\
& +a S\left\{\left([\mathrm{Ag}]_{\mathrm{b}}+[\mathrm{Ag}]_{\mathrm{s}}\right) / 2\right\}+S[\mathrm{Ab}: \mathrm{Ag}] .
\end{aligned}
$$

The molar amount of antibody on the solid surface is

$$
[\mathrm{Ab}]_{\mathrm{t}}=[\mathrm{Ab}]_{\mathrm{f}}+[\mathrm{Ab}: \mathrm{Ag}] .
$$

The unknown variables $\left([\mathrm{Ag}]_{\mathrm{b}},[\mathrm{Ag}]_{\mathrm{g}},[\mathrm{Ab}]_{\mathfrak{f}}\right.$, and [Ab:Ag]) can be calculated from Eqs. [9] to [12] by either the analytical or the numerical method (see below).

With these equations, the concentrations of antigen on the solid surface and bound antigen can be calculated, provided that the independent kinetic variables are experimentally determined.

\section{Determination of Time-Variable Concentrations of Bound Antigen}

Equations [9] to [12] can be reduced to two differential equations by substituting $[\mathrm{Ag}]_{\mathrm{b}}$ and $[\mathrm{Ab}]_{\mathrm{f}}$ in $[9]$ and [10] with [11] and [12]. The two final differential equations are nonlinear and, therefore, difficult to mathematically resolve for two dependent variables (concentrations of antigen on the surface $[\mathrm{Ag}]_{\mathrm{g}}$, and the concentration of the binding complex [Ab:Ag]). However, if we apply two approximations (by assuming pseudo-steady-state antigen concentrations on the surface, and by neglecting the volume of the penetration layer), the equations become mathematically solvable.

The nonlinear equations can also be solved using a numerical method which requires different approximations (see below). We have explored both approaches for 
the kinetic study of antigen-antibody binding at liquidsolid interfaces.

Analytical solution. By definition, a pseudo steady state exists when the diffusion rate of antigen from the bulk solution to the surface is equal to the reaction rate of antigen-antibody binding; no encounter complex or free antigen would have the chance to accumulate in the penetration layer. As we describe in Part II of these investigations, the assumption of a steady-state antigen concentration in the penetration layer is adequate if stirring conditions are used to facilitate the mass transport from the bulk solution to the solid surface (8). Since the influx (Eq. [1]) is equal to the efflux [2], the following equation replaces [9]:

$$
k_{\mathrm{c}}\left([\mathrm{Ag}]_{\mathrm{b}}-[\mathrm{Ag}]_{\mathrm{s}}\right)=k_{\mathrm{on}}[\mathrm{Ab}]_{\mathrm{f}}[\mathrm{Ag}]_{\mathrm{s}}-k_{\text {off }}[\mathrm{Ab}: \mathrm{Ag}] .
$$

We can simplify Eq. [11] by ignoring the volume of the penetration layer (e.g., we have experimentally established that it is $<7 \%$ of the total volume under stirring conditions):

$$
V[\mathrm{Ag}]_{\mathrm{t}}=V[\mathrm{Ag}]_{\mathrm{b}}+S[\mathrm{Ab}: \mathrm{Ag}] .
$$

In Eq. [13], the concentration of antigen at the surface $[\mathrm{Ag}]_{\mathrm{s}}$ can be expressed by $[\mathrm{Ag}]_{\mathrm{b}},[\mathrm{Ab}]_{\mathrm{f}}$, and $[\mathrm{Ab}: \mathrm{Ag}]_{\text {, }}$ and then $[\mathrm{Ag}]_{\mathrm{b}}$ is substituted with Eq. [14]:

$$
\begin{aligned}
{[\mathrm{Ag}]_{\mathrm{s}}=\left\{k _ { \mathrm { c } } \left\{[\mathrm{Ag}]_{\mathrm{t}}\right.\right.} & -\{(1+2 \lambda) /(r \lambda)\}[\mathrm{Ab}: \mathrm{Ag}]\} \\
& \left.+k_{\text {off }}[\mathrm{Ab}: \mathrm{Ag}]\right\} /\left(k_{\mathrm{c}}+k_{\text {on }}[\mathrm{Ab}]_{\mathrm{f}}\right) .
\end{aligned}
$$

$[\mathrm{Ag}]_{\mathrm{g}}$ obtained from [15] is now used in Eq. [10]. To solve $[10],[\mathrm{Ab}]_{f}$ still needs to be replaced and is obtained from [12]. As a result, Eq. [10] becomes

$$
\begin{aligned}
d[\mathrm{Ab}: \mathrm{Ag}] / d t & =N_{\mathrm{Da}_{\mathrm{a}}} k_{\mathrm{c}}[\mathrm{Ag}]_{\mathrm{t}} /\left(1+N_{\mathrm{Da}_{\mathrm{a}}}\right) \\
-\left\{\left\{N_{\mathrm{Da}_{\mathrm{a}}} k_{\mathrm{c}}\{(1+2 \lambda) /(r \lambda)\}+k_{\mathrm{off}}\right\}\right. & \left.\left(1+N_{\mathrm{Da}_{\mathfrak{a}}}\right)\right\}([\mathrm{Ab}: \mathrm{Ag}]) .
\end{aligned}
$$

Equation [16] is now integrated in the range between $[\mathrm{Ab}: \mathrm{Ag}]=0$ and $[\mathrm{Ab}: \mathrm{Ag}]=[\mathrm{Ab}: \mathrm{Ag}]_{t=\mathrm{t}}$, and in the range $t$ $=0$ to $t=\mathrm{t}$. $B / T$ (bound over total antigen) is then determined.

$$
\begin{aligned}
B / T & =\left(S[\mathrm{Ab}: \mathrm{Agg}]_{t=\mathrm{t}}\right) /\left(V[\mathrm{Ag}]_{t=\mathrm{t}}\right) \\
= & \left\{1-\exp \left\{-\left(C+k_{\mathrm{off}}\right) t /\left(1+N_{\mathrm{Da}}\right)\right\}\right\} / \\
& \left(1+k_{\text {off }} C^{-1}\right),
\end{aligned}
$$

where

$$
\mathrm{C}=N_{\mathrm{Da}} k_{\mathrm{c}}\{(1+2 \lambda) /(r \lambda)\} .
$$

Equation [17] is an analytical solution for the variable of interest, $B / T$, which is mathematically expressed as a function of time.
Numerical solution. To circumvent the mathematical approximations in the analytical solution, a numerical method can be used for the theoretical calculations. Numerical methods are algorithms that use only arithmetic and certain logical operations such as algebraic comparisons (15). However, in calculating a function by computer-oriented numerical methods, errors might now be introduced by approximating the solution of a mathematical problem (truncation errors) and in operating a finite number of digits (round-off errors).

For the numerical solution, we use the fourth-order Runge-Kutta method (16) to simultaneously solve the two differential equations [9] and [10] with two supplemental equations [11] and [12]. The purpose of the Runge-Kutta method is to obtain an approximate solution of a system of first-order ordinary differential equations with given initial values.

To calculate the variable of interest, i.e., the ratio of bound over total antigen $(B / T)$ as a function of time, the major steps are:

(a) Scale the variables in Eqs. [9] to [12] $\left([\mathrm{Ag}]_{\mathrm{b}},[\mathrm{Ag}]_{\mathrm{g}}\right.$, [Ab:Ag], $[\mathrm{Ab}]_{\mathrm{f}}$, and $[\mathrm{Ab}]_{\mathrm{t}}$ ) by $[\mathrm{Ag}]_{\mathrm{t}}$.

(b) Scale the time by $\left(a / k_{\mathrm{c}}\right)$.

(c) Define the scaled variables.

(d) Rearrange Eqs. [9] and [10] to introduce the dimensionless groups $\left\{\left(k_{\mathrm{on}}[\mathrm{Ab}]_{\mathrm{t}}\right) / k_{\mathrm{c}}\right\}$ and $\left\{\left(k_{\mathrm{off}} V\right) /\right.$ $\left.\left(k_{\text {on }}[\mathrm{Ab}]_{\mathrm{t}} S\right)\right\}$.

(e) Execute a computer program (e.g., by FORTRAN) for the solution of the equations by the numerical method; i.e., calculate $B / T$ at a given time interval by iterations.

The numerical solution for the problem analyzed in this communication is in Part II and is compared with experimental results.

We have calculated dose-response curves and kinetic binding curves for an immobilized antibody to the steroid hormone progesterone and a radiolabeled and an enzyme-labeled progesterone derivative in microwells by the methods introduced in Part I. The results are compared with experimentally obtained data in Part II of this communication.

\section{ACKNOWLEDGMENTS}

We are indebted to Drs. Leonidas G. Bachas, Richard H. Smith, and Paul A. Craig for critical discussions. This work was supported by the Army Research Office (246655-LS) and a grant from the National Institutes of Health (NICHHD, HD22316).

\section{REFERENCES}

1. Turner, A. P. F., Karube, I., and Wilson, G. S. (1987) Biosensors: Fundamentals and Applications, Oxford Univ. Press, New York.

2. Cooper, J. C., and Hall, E. A. H. (1988) Biomed. Eng. 10, 210-219. 
3. Schramm, W., Yang, T., Paek, S.-H., and Midgley, A. R. (1987) in Proceedings of the Symposium on Sensor Science and Technology (Schumm, B., Jr., Liu, C. C., Powers, R. A., and Yeager, E. B., Eds.), pp. 218-231, The Electrochemical Society, Pennington, NJ.

4. Hlady, V., Lin, J. N., and Andrade, J. D. (1990) Biosens. Bioelectron. 5, 291-301.

5. Schramm, W., and Paek, S.-H. (1991) Anal. Biochem. 196.

6. Stenberg, M., Werthen, M., Theander, S., and Nygren, H. (1988) J. Immunol. Methods 112, 23-29.

7. Berg, O. G., and von Hippel, P. H. (1985) Annu. Rev. Biophys. Biophys. Chem. 14, 131-160.

8. Trurnit, H. J. (1954) Arch. Biochem. 51, 176-199.

9. Welty, J. R., Wilson, R. E., and Wicks, C. E. (1976) Fundamen- tals of Momentum Heat and Mass Transfer, p. 578-607, Wiley, New York.

10. Wiegel, F. W., and DeLisi, C. (1982) Am. J. Physiol. 243, R475R479.

11. Berg, O. G. (1985) Biophys. J. 47, 1-14.

12. Shoup, D., Lipari, G., and Szabo, A. (1981) Biophys. J. 36, 697714.

13. Frey, S., and Tamm, L. (1990) Biochem. J. 272, 713-719.

14. Eddowes, M. J. (1987) Biosensors 3, 1-15.

15. Carnahan, B., Luther, H. A., and Wilkes, J. O. (1969) Applied Numerical Methods, pp. 319-329, Wiley, New York.

16. Romanelli, M. J. (1960) in Mathematical Methods for Digital Computers (Ralston, A., and Wilf, H. S., Eds.), pp. 110-120, Wiley, New York. 